\title{
La responsabilité historique de Vera Feyder ${ }^{1}$
}

\author{
Historical responsibility of Vera Feyder
}

\author{
Judyta Zbierska-Mościcka \\ Université de Varsovie \\ e-mail: j.zbierska-moscicka@uw.edu.pl
}

\begin{abstract}
In her writing, Vera Feyder, representative of the tribe called by the historians of Belgian literature generation of identity, the question of identity is inextricably linked with the experience of World War II. The memory of childhood marked by the martyrdom of his father and the deep misery and loneliness, in which the author and his mother lived in the wartime, decide on the content and meaning of her writing. A sense of responsibility for other people, all resistance to the injustice, disagreement with indifference, memory of the past - it is a kind of code, which, according to Feyder, should apply a modern writer.
\end{abstract}

Keywords: Belgian Literature, Vera Feyder, Responsibility, History, Identity

«Les lieux se vident/ les mots se perdent/ dans l'infiniment grand dommage que le temps/ et l'espace ourdissent incessamment contre eux » (2006b : 353), écrit Vera Feyder dans son poème « Histoire », publié dans le recueil Le fond de l'être est froid de 1995. L'auteur appartient à la génération d'écrivains qui, étant nés pendant la Seconde Guerre mondiale, se reconnaissent à la fois porteurs et passeurs d'une mémoire lacunaire certes, mais fondatrice d'une manière d'être au monde, d'une manière d'être soi. Benoît Denis et Jean-Marie Klinkenberg appellent cette génération « identitaire» $(2005: 248)$, dont le nom doit renvoyer au mouvement belgitude qui fait de l'affirmation identitaire l'essence de ses préoccupations. Cette affirmation identitaire est censée se nourrir de la mémoire, de l'Histoire que l'on a voulu écarter

\footnotetext{
${ }^{1}$ Projet financé par les fonds du Centre National de la Science, alloués par le biais de décision DEC-2013/09/B/HS2/01168.
} 
en Belgique, dont on n'a pas voulu faire partie ni reconnaître l'importance. Trois grands thèmes littéraires - mémoire, Histoire, identité - se retrouvent, chez Feyder, conjugués à celui de l'espace, l'espace de l'oubli et l'espace d'une mémoire reconquise, et jouent dans son œuvre un rôle crucial, fondateur de l'écriture.

L'œuvre de Feyder, poétique, dramatique ou narrative, émerge au début des années 60 qui voient apparaître, successivement, ses premiers poèmes (1961, le recueil Le Temps démuni) et sa première nouvelle (1965, Un jaspe pour Liza) que suivent, dans les années 70, son premier roman (La Derelitta, 1976) et sa première pièce de théâtre (1978, Emballage perdu). Elle naît d'une perte, d'un vide que laisse la disparition de son père, emporté, en 1942, à Auschwitz, suite à une dénonciation de l'un de ses collègues. Maurice Federman n'en revient pas, mort probablement lors du transport d'Auschwitz à Buchenwald, quelques mois avant la fin de la guerre. L'absence du père, due à l'ignominie humaine que la fille dénoncera en des mots amers dans l'ouvrage consacré à Liège, sa ville natale, en $1992^{2}$, plonge la fille et sa mère dans une misère éprouvante qui réduit la mère à un travail épuisant pour la subsistance, et Vera à une errance de plusieurs années, entre sanatoriums et homes pour enfants démunis. Cette expérience la marque à vie, elle ne s'en remettra jamais. Le sentiment d'abandon, de délaissement, celui enfin de rester aux marges de la vie, Vera Feyder les communique à ses protagonistes. Ceux-ci, pareils à des fantômes, sont des déshérités, des orphelins arrachés à un milieu protecteur, déracinés souvent par les événements dont ils ne sont pas responsables. Ils se cherchent, se perdent, se retrouvent, dans une errance qui semble infinie, marqués par cette rupture initiale qui les a propulsés vers un destin inconnu.

L'image de la guerre, on la retrouve aisément dans le souvenir d'une enfance carcérale, enfermée dans des dortoirs froids et impersonnels, dont la plupart des protagonistes de Feyder a fait l'expérience. De La Derelitta (1976) à La belle voyageuse endormie dans la brousse (2003), les personnages se débattent dans des intérieurs étouffants, étrangers, passagers, qui ne leur appartiennent pas. Le motif de l'enfermement, hérité par Feyder de son passé d'orpheline, est peut-être aussi une manière d'évoquer le sort carcéral et incertain de son propre père. Le souvenir de celui-ci est exprimé en toutes lettres dans le premier roman de Feyder, dont le titre, La Derelitta, dit l'état d'esprit qui par ailleurs n'est pas uniquement celui d'Eva, la protagoniste du roman. L'image de son père agonisant à Auschwitz se superpose ici à d'autres qui concentrent sa douleur d'être, trop longtemps contenue.

Le thème de la guerre apparaît dans d'autres textes aussi. Dans le récit Un jaspe pour Liza, l'atrocité de la guerre surgit au travers de l'histoire d'une amitié d'un

\footnotetext{
${ }^{2}$ V. Feyder, Liège, Seyssel, Champ Vallon, 1992, p. 102 : «Est-ce pour cela que je ne puis, des dizaines d'années après, y revenir sans un serrement de cœur?, se demande-t-elle dans son essai autobiographique Liège. Sans qu'un relent de vase et d'infamie, jamais épongé, ne me lève le cœur à son approche ? Ni descendre d'un train sans penser que de cette gare des Guillemins, si changée pourtant dans son architecture, ses couleurs, ses odeurs, il est parti, lui, pour une destination alors inconnue, dont on sait maintenant, et chaque jour davantage, ce qu'elle était ? ».
} 
homme mûr, Edward Barnem, et d'une jeune fille, Liza, délaissée momentanément par ses parents «fuyant les pogroms de leur Pologne natale » (2008a : 17). Liza ayant rejoint enfin ses parents en Belgique, Barnem lui envoie un cadeau pour son anniversaire, « un jaspe sanguin de la couleur de ses yeux » (2008a : 21). Quelques années après, à New York, ayant déjà appris la disparition de Liza et de sa famille déportée vers les camps, il retrouve le pendentif à jaspe sur le cou d'une jeune Allemande. Ses parents veillent à la bonne éducation de leur fille et l'envoient à Barnem pour les leçons de français. Ce dernier lui arrache le pendentif, la fille disparaît effrayée pour se réfugier chez sa tante, Barnem est arrêté, soupçonné d'avoir tué la fille. Quelque temps après il se suicide dans la prison.

Dans La bouche de l'Ogre, un récit de 2003, Feyder met en scène une femme, Alba, qui, lors d'une randonnée à la montagne, quelque part en Autriche, renonce à aider une femme, suspendue au bord d'un gouffre et hurlant en allemand des appels insistants de secours. Ayant entendu dans ces appels des ordres articulés « dans une langue qui sera toujours, pour [elle], celle des bourreaux, des officiants serviles de la nuit et du brouillard » (2008c : 114), elle n'a pas su résister à l'emprise de la haine qu'elle portait depuis que les siens avaient disparu derrière les barbelés.

Ainsi, le thème de la mémoire de la guerre, d'un souvenir invariablement douloureux mais nécessaire, parcourt l'œuvre de Feyder de part en part. Il s'incarne notamment dans l'individu ravagé par la nostalgie d'un passé brisé, mais prend aussi d'autres déguisements dont on verra tout à l'heure l'exemple.

Nous retiendrons pour la présente analyse le roman Caldeiras, publié en 1982, donc à l'époque de l'interrogation identitaire suscitée, entre autres, par la publication, en 1976, du dossier "L'Autre Belgique » par Pierre Mertens et Claude Javeau, dans les Nouvelles littéraires ${ }^{3}$. Il s'agit bien du moment qui dit la nécessité d'un retour du refoulé, le retour de l'Histoire, et qui articule ainsi l'importance d'un certain engagement, déficitaire jusqu'ici. C'est aussi l'époque où les représentants de la génération identitaire s'imposent sur la scène littéraire belge pour, en un léger décalage par rapport à la France, y faire figure d'intellectuels impliqués dans un débat critique sur la société :

Le rôle d'intellectuel engagé, qui semblait depuis la Libération être une spécialité française, refait surface en Belgique. [...] contemporains de la décolonisation et de la réforme de l'État, ces intellectuels ne vivent pas le rapport assuré à l'universalité qui était celui de leurs aînés ; leur discours sera moins celui des grandes certitudes et du prophétisme politique qu'une interrogation critique sur le rôle et la place de l'intellectuel dans la société et sur la possibilité pour lui d'exprimer une forme quelconque de radicalité (2005: 250).

\footnotetext{
${ }^{3}$ D'autres publications nourrissent, en effet, le débat, dont il faut sans doute citer « Littérature et fonctionnement idéologique en Belgique francophone " (dans La Belgique malgré tout, $\mathrm{n}^{\text {os }} 1-4$ de la Revue de l'Université de Bruxelles, 1980) et Balises pour l'histoire de nos lettres (1982) de Marc Quaghebeur, ainsi que Lettres françaises de Belgique. Mutations. Entretiens de Paul Emond (1980).
} 
Denis et Klinkenberg citent à l'occasion les noms de Pierre Mertens, de Conrad Detrez, de Jean Louvet ou de René Kalisky. Vera Feyder, on 1'a vu, n'a pas attendu le mouvement de belgitude pour faire de l'Histoire le levier de ses œuvres. Sans compter le recueil Le Temps démuni, dédicacé, en 1961, au père défunt et à la mère « infiniment proche », la nouvelle Un jaspe pour Liza, accueillie par Simone de Beauvoir dans Les Temps modernes, en 1965, témoigne déjà d'une posture qui sera celle de Vera Feyder ${ }^{4}$. Engagement et responsabilité, participation et présence, mémoire et résistance seront les idées-clefs de cette œuvre nourrie par l'expérience personnelle de l'auteur, expérience que celle-ci se proposera de dépasser. Il s'agira donc ici de voir comment la mémoire de la guerre, l'origine et l'aliment principal de l'écriture feyderienne, se cristallise en une éthique d'artiste, une attitude morale et intellectuelle inséparable de l'acte créateur.

«Prendre la plume, c'est aussi une façon de prendre les armes sans faire couler autre chose que de l'encre » (2000), affirme Feyder, mais cette attitude ne date pas de ce moment-là. Réagir à une réalité révoltante semble à l'écrivain un devoir de première importance, que le statut d'artiste lui permet de réaliser avec l'espoir d'un retentissement susceptible d'être fructueux. En 1988, dans un entretien avec Michel Voiturier, elle avoue qu'elle « écri[t] pour sauver l'esprit, l'âme, la conscience face à cette avalanche de mots et d'images qui [...] nous engloutissent au lieu de nous éclairer ». Elle est convaincue que «l'écrivain, face aux consciences collectives manipulées, doit sortir du rang et espérer qu'au milieu de cette cacophonie de dictature, il aura des suiveurs, des gens qui tentent aussi de sauver l'esprit » (1988). Sa participation à un des numéros des Temps modernes, en 1965, ne semble pas ainsi uniquement l'effet d'une coïncidence heureuse mais l'expression d'une conviction profonde. « [...] il n’y a pas de littérature innocente » (1998 : 39), dit bien Jean-Paul Sartre dans La responsabilité de l'écrivain ${ }^{5}$; l'artiste, l'écrivain et le romancier a fortiori a cette possibilité d'objectiver les choses et de les soumettre ainsi à la réflexion (1998: 19). « Nommer le désespoir, c'est le dépasser» (1951: 314), écrit Camus dans L'homme révolté. Vera Feyder semble, en effet, poursuivre cette voie : nommer, mettre en évidence, prendre la défense de ceux qui ne sont pas ou ne sont plus capables de se défendre ( hommes et bêtes ${ }^{6}$ ), dénoncer, rendre compte et régler les comptes, ne jamais rester à côté, ne rien passer sous silence. L'écriture, c'est pour elle la liberté de dire, de faire, de "se mettre hors-la-loi du monde » (1977), c'est un privilège dont elle se sert d'autant plus volontiers qu'elle considère son enfance comme une période «bâillonnée » où elle n'a pas eu l'audace d'exprimer, voire de crier son désespoir.

\footnotetext{
${ }^{4}$ La nouvelle est, en effet, publiée dans le numéro 227 de la revue Les Temps modernes, en avril 1965.

${ }^{5}$ C'est sous ce titre que paraît, en 1998, le texte de la contribution de Sartre à la Conférence générale de l'Unesco qui s'est déroulée à Paris, en novembre 1946.

${ }^{6}$ Dédicace à la nouvelle Nul conquérant n'arrive à temps de 1978 : «aux déracinés, opprimés, torturés - hommes et bêtes » (2008b : 47).
} 
« Si je veux vivre/ il me faudra réécrire la vie » (2006a : 39), dit Feyder dans un poème du recueil Le Temps démuni, et le roman Caldeiras le lui permet, car c'est un roman qui laisse se dérouler les fils de plusieurs vies dont on suit les itinéraires tantôt brisés, tantôt repris. Les destins s'enchevêtrent inlassablement, les personnages foisonnent, unis les uns aux autres dans leur condition d'errants en une quête inlassable de l'identité. Deux individus pourtant jouent dans le roman un rôle bien distinct : à savoir Tina et Nat qui semblent figurer des alter ego de l'écrivain ou d'incarner deux phases ou deux aspects de son existence. On remarquera par ailleurs la ressemblance des prénoms, l'un étant un anagramme presque parfait de l'autre, ce qui ne nous semble pas fortuit.

Tina est une jeune femme, orpheline et solitaire, qui gagne sa vie dans un atelier de couture et qui rêve à s'échapper de cette existence qu'elle trouve imparfaite. De brefs épisodes de dépaysement que lui offrent les visites au cinéma ou à l'opéra, ses fugues habituelles, ne font que renforcer le sentiment d'étouffement. Car elle étouffe dans ce Liège labyrinthique qu'elle sillonne lors de ses flâneries quotidiennes, piégée par cette ville bien connue et pourtant d'une certaine façon ennemie. Comme le Liège de l'année 1942 qui est devenu la scène du drame familial :

Est-ce pour cela que je ne puis, des dizaines d'années après, y revenir sans un serrement de cœur?, se demande Vera Feyder dans son essai autobiographique Liège. Sans qu'un relent de vase et d'infamie, jamais épongé, ne me lève le cœur à son approche ? Ni descendre d'un train sans penser que de cette gare des Guillemins, si changée pourtant dans son architecture, ses couleurs, ses odeurs, il est parti, lui, pour une destination alors inconnue, dont on sait maintenant, et chaque jour davantage, ce qu'elle était ? (1992 : 102)

Les déambulations diurnes ou nocturnes de Tina, à travers Liège (espace de l'oubli et de l'effacement d'un passé intraitable), déambulations très nombreuses et emblématiques d'une nécessité de s'épanouir en dehors de ce cadre oppresseur, disent la révolte ou, comme l'appelle Feyder dans le roman, l'insurrection contre une vie étroite, une vie en marge, contre une certaine passivité (Feyder parle, en effet, d'une «enfance bâillonnée » ou d'une «jeunesse potencée » qui l'a laissée sans voix ni sans la possibilité d'agir). "Le monde appartient aux insurgés » (2004) confie Feyder à Jean-Pierre Verheggen dans une interview. Ne pas oublier, perpétuer la mémoire des disparus ou celle de l'impensable est, en effet, une forme de révolte que Feyder comprend comme Albert Camus : «Dans la révolte, l'homme se dépasse en autrui et, de ce point de vue, la solidarité humaine est métaphysique » $(1951: 29)$, lisons-nous dans L'homme révolté. La révolte de Tina, bien que très personnelle et focalisée sur une réussite personnelle, est dirigée vers l'autre. C'est à travers l'autre, un homme aimé et qui l'aimera, qu'elle peut dépasser son destin et en construire un nouveau, en dépit du monde.

Tina est l'un des nombreux masques de Feyder, un masque qui en cache d'autres, car l'hérö̈ne se présente tantôt comme Augustina (donc Tina) Mars, tantôt 
comme Pearl Parradine, tantôt, et c'est son véritable identité, comme Mila Orkiavjen, une Juive d'origine polonaise (comme le père de Feyder) dont les parents ont disparu à Auschwitz. Nat est un déguisement de plus qui, sous les traits d'un correspondant de guerre revisitant en mémoire ses « terrains de chasse », développe une réflexion sur la relation de l'homme à l'Histoire. Il apparaît lui-même sous différents pseudonymes. Qu'il se nomme Johnathan Cadwallader Mardochée, John Cadwell, Doc Mardoch ou Jessie Haime, le protagoniste nouvellement auréolé du Prix Gulliver (Pulitzer ?), s'enfuit de l'hôtel Walldorf à New York où il est fêté, pour repenser sa vie de, comme il dit, " grand rapporteur d'ignominies et de crimes impunis » (2001 : 420). Afin de refaire mentalement son parcours, ce qui s'avère au bout du compte un itinéraire d'expiation, il choisit, chose intéressante, Bruxelles, et ceci «parce qu'on s'y sentait, comme nulle part ailleurs, étranger» (2001: 26). «On [y] passe pour ce qu'on paraît, jamais pour ce qu'on est. Cela facilite les choses, en les rendant aussi inconsistantes, aussi creuses que possible » (2001:27), dit Nat dans une lettre où il confie ses réflexions à une certaine Cordelia, son ancien amour. Bruxelles devient ici l'espace neutre où s'accomplit la lente métamorphose de Nat. Baignée dans la pluie, la ville accueille les flâneries dubitatives de l'homme acculé à faire un vide en soi, conscient d'un coup d'avoir commis le crime de la « non-ingérence ». Ayant parcouru le monde entier, des «bourbiers d'Indochine » aux «plus bas quartiers des Amériques et de l'Inde », ayant capté avec le regard impersonnel et impitoyable du caméra «les enfants drogués » et « les prostituées de Bogota » (2001: 205-206), soit tous ces «hauts lieux de la misère et de l'humiliation » (2001: 206), il prend conscience qu'il n'était dans tout cela qu'un pauvre « exécuteur de commandes » travaillant afin d'assouvir l'avidité marchande des grands manipulateurs des consciences. La médiation de l'œil de l'appareil photographique lui a, en effet, enlevé la vue et a amené « l'insensibilisation et le refroidissement progressifs de tout son être » (2001 : 221). Deux circonstances précipitent l'évolution qu'il subit. La première est la rencontre d'Adam Feneczski, un majordome de l'hôtel Walldorf qui l'aide à s'éclipser, mais qui est surtout un rescapé du ghetto de Varsovie. Toute sa famille, sa femme et quatre enfants, avaient été victimes des expérimentations médicales des nazis. Il s'était désormais consacré à poursuivre le bourreau de sa famille qu'il a retrouvé quelque part en Amérique du Sud où il a accompli sa vengeance, ce dont il fait part dans une brève lettre à Nat : « Ne cherchez plus, j'ai trouvé » (2001: 429).

La deuxième circonstance est le voyage commémoratif vers les camps de concentration de Dachau, Auschwitz et Buchenwald, auquel sont invités les reporters et journalistes. Indifférent devant le spectacle de l'ignominie humaine, armé de sa caméra infaillible qui devait mémoriser les images de ces lieux désolés, il s'est alors rendu compte du fait que la mémoire de ces lieux devait être autre chose que des kilomètres de clichés rangés dans des tiroirs. "Sa mémoire, si mémoire il y avait, gisait indéfectiblement là, dans des boîtes, des rouleaux, des archives - répertoriés 
dans des agences, classés » (2001: 206). Et pourtant, il était là, lui, l'homme, le témoin, celui qui a vu mais qui n'a pas réagi. C'est en cela, en effet, que consiste son crime qu'il doit expier, à « avoir su et [à] n'être à aucun moment intervenu » (2001 : 432). Le geste qu'il accomplit en guise d'expiation est emblématique : il ouvre la fenêtre de sa chambre au quinzième étage d'un hôtel et «suicid[e] un à un ses appareils. John Cadwell n'[est] plus » (2001: 435).

Le roman Caldeiras se situe presque au milieu de la vie littéraire de Vera Feyder. Il est une œuvre de maturité (Feyder est une quadragénaire au moment de la publication), préparée par de longues années de méditation dont les sédiments en ont formé la trame et le sens, issue d'une errance inachevée qui a son commencement en 1942, à Liège. Il tombe au milieu de la vie artistique de Feyder et au milieu d'une nouvelle interrogation identitaire qui met les Belges en face d'eux-mêmes, en essayant de les rendre responsables de leur belgité, les uns devant les autres. Il voit le jour en 1982, l'année de la publication des Balises pour l'histoire de nos lettres où Marc Quaghebeur dénonce le péché de la déshistoire qui rend les Belges étrangers à leur propre pays. Caldeiras semble être une réponse à cette dénonciation, une réponse discrète, très personnelle, déguisée. C'est un roman de la mémoire et sur la mémoire qui veut dire responsabilité et présence. Et pour terminer avec une nouvelle allusion à l'œuvre poétique de Feyder, on dira que c'est un roman écrit, comme le titre de l'un des recueils le dit contre toute absence. 


\section{BIBLIOGRAPHIE}

Camus, A. (1951). L'homme révolté, Paris : Gallimard.

Denis, B., Klinkenberg, J.-M. (2005). La littérature belge. Précis d'histoire sociale, Bruxelles : Labor.

Dessambre, D. (2000). «Prendre la plume, prendre les armes », Gazette de Liège, le 16 novembre. Feyder, V. (1992). Liège, Seyssel : Champ Vallon.

- (2001). Caldeiras (1982). Bruxelles : Ancrage.

- (2006a). «Si je veux vivre», in Le Temps démuni (1961), in Contre toute absence. Poèmes (1960-2003), Châtelineau (Belgique) : Le Taillis Pré.

- (2006b). «Histoire», in Le fond de l'être est froid (1995), in Contre toute absence. Poèmes (1960-2003), Châtelineau (Belgique) : Le Taillis Pré.

- (2008a). «Un jaspe pour Liza» (1965), in Ô humanité !, Bruxelles : Le Grand Miroir.

- (2008b). «Nul conquérant n'arrive à temps » (1978), in Ô humanité !, Bruxelles : Le Grand Miroir.

- (2008c). « La bouche de l'ogre » (2003), in Ô humanité !, Bruxelles : Le Grand Miroir.

Jacques, J.-L. (1977). Interview avec Vera Feyder diffusée dans l'émission Actuel III sur RTBF.

Disponible dans le dossier de presse consacré à Vera Feyder, aux Archives et Musée de la Littérature à Bruxelles, sous la cote MLCP 696-706.

Sartre, J.-P. (1998). La responsabilité de l'écrivain, Verdier.

Verheggen, J.-P. (2004). Interview avec Vera Feyder enregistrée sur un CD in Littérature au présent. Cinquante et un, Bruxelles : la Maison d'à côté.

Voiturier, M. (1988). Interview in Courrier de l'Escaut, le 2 février. 